\title{
MAPUDUNGUWELAYMI AM? ‘ACASO YA NO HABLAS MAPUDUNGUN?’ ACERCA DEL ESTADO ACTUAL DE LA LENGUA MAPUCHE
}

\author{
Fernando Zúñiga
}

El presente artículo esboza una evaluación tentativa de la vitalidad etnolingüística actual de la lengua mapuche en Chile en el marco de los parámetros propuestos por Landweer (2000) y sobre la base de los resultados de la encuesta específica realizada por el Centro de Estudios Públicos en 2006. La principal conclusión es que el mapudungun parece encontrarse en un estado de precariedad sociolingüística, y que sólo políticas públicas e iniciativas privadas que conduzcan a una revitalización efectiva y eficaz en el corto plazo lograrán salvar a la lengua de la extinción inminente.

Fernando ZúñIga. Ingeniero Comercial Economista de la Pontificia Universidad Católica de Chile, Magíster y Doctor en Lingüística Comparada de la Universidad de Zúrich, Suiza. Se ha desempeñado como docente e investigador en el área de la lingüística comparada en diferentes universidades (Zúrich, Leipzig, Mánchester, La Trobe, Oregón) y como investigador del Centro de Estudios Públicos. Actualmente es docente e investigador en el Departamento de Lingüística General de la Universidad de Zúrich.

N. del E.: Sobre los resultados de la encuesta mapuche realizada por el CEP (2006), véanse también en esta edición los trabajos de Eduardo Valenzuela, Ignacio Irarrázaval y M. de los Ángeles Morandé, Aldo Mascareño, Rolf Foerster y Sonia Montecino, Juan M. Ossio, así como el comentario de Jorge Larraín al artículo de Aldo Mascareño y la presentación de Carolina Segovia y Lucas Sierra. 


\section{Introducción}

I

ncluso un conocimiento superficial de la historia del continente americano permite concluir que los últimos quinientos años no han sido especialmente favorables al desarrollo de las lenguas indígenas. El mapudungun o lengua mapuche de Chile y Argentina no ha sido una excepción: por un lado, en las primeras páginas de la más temprana descripción de la lengua mapuche — Valdivia (1606)—, su autor decía que

[e]n todo el Reino de Chile no ay mas de esta lengua que corre desde la Ciudad de Coquimbo y sus terminos, hasta las yslas de Chilue y mas adelante, por espacio casi de quatrocientas leguas de Norte a Sur que es la longitud del Reyno de Chile, y desde el pie de la Cordillera grande neuada, hasta la mar, que es el ancho de aquel reyno, por espacio de veynte leguas.

Sin embargo, en el prefacio de su gramática mapuche de hace poco más de un siglo, Félix José de Augusta aseveraba que "es fácil prever que el idioma indígena se hablará en Chile de aquí a unos cien años” (1903: viii). A comienzos del siglo XX, Pascual Coña (2002: 25) afirmaba:

Fewla kalewetuy mongen; tüfachi weche mütewe wingkatuingün; allwe ngoymarpuingün taiñ küpal ñi ngülam ka ñi dungu; kalli rupape kiñe mufü tripantu, feymew epe kimwerpulayay ñi mapudungun engün.

'En nuestros días la vida ha cambiado; la generación nueva se ha chilenizado mucho; poco a poco ha ido olvidándose del designio y de la índole de nuestra raza, que pasen unos cuantos años y casi ni sabrán ya hablar su lengua nativa’.

En efecto, un visitante del Cono Sur a comienzos del siglo XXI puede llevarse la impresión de que, si bien parte importante de la población es mestiza, la cultura indígena tiene un rol tan marginal que la hace virtualmente invisible, y el mapudungun no está presente en los medios de comunicación ni en la calle de ninguna ciudad de la región. Al igual que en vastas extensiones de Norteamérica y otras regiones de Sudamérica, los habitantes del territorio creen tener buenas razones para pensar que la lengua indígena ha desaparecido dejando vestigios aislados en el folclor, la toponimia y la patronimia.

Afortunadamente, el sombrío pronóstico de Augusta y Coña no se ha cumplido: afirmar que la cultura y la lengua indígenas han desaparecido 
es todavía indiscutiblemente un error. Los historiadores, sociólogos, antropólogos y lingüistas continúan documentando y profundizando el conocimiento que se tiene acerca de ellas - tanto en su dimensión diacrónica, con el fin de comprender mejor los procesos históricos que las han moldeado, como en su dimensión sincrónica, arrojando luz sobre sus contenidos y su dinámica actuales y sus relaciones con la cambiante e influyente cultura hispanochilena- . No obstante, el diagnóstico superficial respecto de la notoria disminución de importancia y vitalidad del mapudungun parece seguir siendo válido en cierto sentido, y la pregunta que nos interesa en este contexto es: ¿cuál es la pujanza del mapudungun hoy?

El presente artículo busca delinear al menos algunas respuestas a esta significativa pregunta sobre la base de los resultados de la encuesta elaborada y realizada por el Centro de Estudios Públicos en 2006 (CEP, 2006). Para ello, la sección 1 esboza algunos parámetros que permiten evaluar la vitalidad de una lengua determinada (así como, hasta cierto punto, de la cultura que la alberga y a la cual sirve de instrumento). La sección 2 examina los datos arrojados por la encuesta a la luz del marco conceptual introducido en la sección 1 . Por último, la sección 3 presenta las conclusiones que se pueden sacar desde una perspectiva sociolingüística y menciona algunas cosas que pueden hacerse si el objetivo es aumentar la vitalidad del mapudungun.

\section{La vitalidad etnolingüística}

Aun cuando la preocupación por la inminente extinción de un gran número de las lenguas del mundo por parte de los estudiosos y de quienes están involucrados con el diseño y la práctica de políticas lingüísticas no es reciente, la última década ha visto surgir un interés particularmente grande a nivel mundial por el tema; algunos de los trabajos, recientes o por aparecer, que dan cuenta de ello son Crystal (2002), Nettle y Romaine (2002), Brenzinger (2007) y Moseley (2007). El reverso lógico de las razones que llevan a la extinción de algunos idiomas es el conjunto de causas de que otras lenguas gocen de buena salud, y de esto se preocupa un creciente volumen de literatura que estudia los casos fallidos de revitalización lingüística, así como también los exitosos; baste mencionar aquí estudios sinópticos y teóricos recientes como Grenoble y Whaley (1998, 2005), Fishman (2001), Hinton y Hale (2001) y Tsunoda (2005). En términos muy sucintos, baste decir aquí, a partir de las experiencias recogidas y las observaciones hechas en la literatura, que 
(a) ha habido extinción de lenguas en diferentes períodos de la historia, pero que actualmente dicho proceso ha alcanzado proporciones considerables a nivel mundial;

(b) es posible elaborar un diagnóstico acerca de cuán vital es una lengua en un momento y un lugar determinados; y

(c) es posible diseñar e implementar políticas de revitalización exitosas si las condiciones sociológicas, antropológicas, históricas y políticas son propicias.

Basada sobre su propia experiencia con lenguas de Papúa Nueva Guinea, así como sobre aquella de otros investigadores en esa misma región y otros sitios del mundo durante las últimas tres décadas, Landweer (2000) propone los siguientes ocho indicadores de vitalidad etnolingüística que han resultado útiles para pronosticar la viabilidad de una lengua determinada en una región dada en el corto, el mediano y el largo plazo. Naturalmente, la interacción entre los diversos factores capturados por dichos indicadores puede ser distinta en diferentes partes del mundo, y también variar a lo largo del tiempo:

1) Posición relativa en el continuum urbano-rural;

2) ámbitos de uso de la lengua;

3) diglosia y code switching;

4) dinámica poblacional y de grupo;

5) distribución de los hablantes al interior de sus redes sociales;

6) fuerza identitaria de la comunidad lingüística;

7) prestigio de la lengua; $y$

8) acceso a una base económica estable y aceptable.

A continuación explicamos brevemente cada uno de estos parámetros (cada uno con cuatro valores o posiciones a lo largo de una escala), incluyendo información general acerca de dónde se ubica el mapudungun —mejor dicho, donde puede ubicarse el mapudungun hablado por diferentes grupos en diferentes sitios de Chile-.

La posición relativa en el continuum urbano-rural no sólo se refiere a si la lengua en cuestión se habla en un entorno rural o urbano — se ha observado que una lengua minoritaria o minorizada tiene menos posibilidades de sobrevivencia en una ciudad-, sino también al tipo de conexiones que puedan existir entre áreas urbanas y rurales relevantes, así como al uso que los hablantes hagan de dichas conexiones. Por ejemplo, puede que la lengua minoritaria se hable en una zona rural, pero las vías de acceso a una ciudad cercana sean buenas y los hablantes hagan uso frecuente de ellas 
con el objeto de participar en el comercio o las actividades productivas ofrecidas por la urbe. De este modo, el continuum urbano-rural puede articularse en torno a cuatro posiciones que probablemente reflejen una vitalidad creciente: (i) la lengua amenazada se encuentra en la ciudad, (ii) la lengua amenazada se encuentra en la periferia de algún centro urbano, con acceso fácil a él, (iii) el acceso de los hablantes de la lengua amenazada al centro urbano es difícil o marginal, y (iv) la lengua minoritaria se encuentra en una zona remota, ya sea por la distancia de las ciudades o por los malos accesos. Tanto la información censal como la observación directa permiten concluir que hablantes del mapudungun se encuentran en cada una de estas cuatro situaciones; parece razonablemente claro que el porcentaje de hablantes urbanos - (i) y (ii) — va en aumento.

La vitalidad de una lengua será mayor mientras más ámbitos de uso tenga. Se ha observado repetidamente y en todos los continentes que el último ámbito en ser abandonado es el familiar o doméstico, con lo cual los cuatro valores de este parámetro son: (i) la lengua minoritaria se utiliza sólo en el ámbito doméstico, e incluso allí en combinación con un idioma externo, (ii) la lengua minoritaria se utiliza en el ámbito doméstico y en "eventos culturales” (fiestas, ceremonias, etc.), pero también se la combina con un idioma externo, (iii) la lengua minoritaria se usa en el ámbito doméstico, cultural y social, y (iv) la lengua minoritaria se utiliza en todos los ámbitos de la vida comunitaria. Los datos de la encuesta, presentados y comentados en la próxima sección, arrojan alguna luz sobre el valor empírico de este parámetro.

Diglosia es el término empleado en sociolingüística para denominar una situación en la cual una comunidad lingüística utiliza dos variedades lingüísticas — sean éstas registros de una misma lengua, dialectos o lenguas diferentes - según un patrón relativamente rígido de diferentes funciones sociales: por ejemplo, una comunidad puede usar una lengua global en el ámbito de la religión, la educación y eventos festivos u oficiales, y una lengua local en el ámbito del hogar y el comercio; la referencia clásica es Ferguson (1959). Code switching (CS) significa que miembros de una comunidad lingüística utilizan una variedad lingüística o "código" en algunos ámbitos de modo variable y menos rígido que en la situación diglósica; se puede hablar incluso de CS entre oraciones —o sea, un hablante dice algunas oraciones en una lengua y otras en otra, durante el mismo discurso acerca del mismo tema y en la misma situación- y al interior de oraciones — es decir, parte de una oración, ya sea algunas palabras o unidades mayores, están en una variedad lingüística y el resto en otra-. Los cuatro valores de este parámetro son: (i) CS frecuente, (ii) CS poco frecuente, 
(iii) bilingüismo o diglosia estables, y (iv) mayoría de la población monolingüe en la lengua minoritaria. Los datos de la encuesta no son buenos indicadores de este parámetro, y un estudio sociolingüístico detallado es la herramienta deseable en este sentido; aun así, la información recogida por parte del autor sugiere que la mayoría de los hablantes se encuentra en las situaciones (i) o (ii).

En cuanto al tercer parámetro mencionado, la dinámica poblacional y de grupo, una de las observaciones recurrentes es que una comunidad lingüística debe contar con una masa crítica para ser viable, si bien dicha masa crítica puede variar grandemente de un lugar a otro, o aun a lo largo del tiempo. No obstante, no sólo el número relativo de hablantes de una lengua minoritaria es relevante: también lo es el comportamiento de inmigrantes que lleguen a la zona tradicionalmente asociada con la comunidad lingüística, el cual está relacionado con el factor de la masa crítica de modo complejo. Así, las posiciones de este parámetro son: (i) los inmigrantes mantienen su idioma e insisten en que quienes interactúen con ellos también lo hablen, sin desarrollar siquiera una competencia pasiva en la lengua minoritaria, (ii) los inmigrantes interactúan con los nativos en una lengua distinta de la propia y de la minoritaria (p. ej., una lingua franca como el inglés), (iii) los inmigrantes comprenden la lengua minoritaria pero no la hablan, interactuando con los nativos en su propia lengua o en una lingua franca, y (iv) los inmigrantes son bilingües y utilizan la lengua minoritaria activamente. Si bien en el pasado, así como actualmente en algunas zonas relativamente remotas de las regiones VIII y IX, parece haber habido casos numerosos de (iv), actualmente la situación (i) parece ser absolutamente dominante. Algunos datos de la encuesta avalan esta observación.

Las redes sociales en las cuales viven los hablantes de una lengua son más densas en la medida en que las diferentes personas están relacionadas unas con otras (vía parentesco, vecindad, oficio, etc.), y las relaciones son múltiples si, por ejemplo, dos personas dadas están relacionadas de más de una forma — son vecinas y a la vez primas, y trabajan juntas(Landweer, 1991). Las posiciones de la escala para este parámetro, que toman en cuenta tanto estos dos aspectos como la interacción con redes externas a la comunidad lingüística, son: (i) los individuos son relativamente independientes en redes poco densas y no múltiples, (ii) existen sistemas de redes densas, pero están abiertos hacia el exterior, donde hay hablantes que no utilizan la lengua minoritaria, (iii) existen sistemas de redes densas con poca necesidad de utilizar una lengua diferente de la minoritaria en la interacción con redes externas, y (iv) las redes sociales son densas y múltiples, y todas las necesidades comunicacionales se satisfacen a través de la len- 
gua minoritaria. La encuesta no arroja información respecto de este parámetro, y no soy un antropólogo con conocimiento amplio de este tipo de variables en el mundo mapuche rural y urbano, por lo cual evito pronunciarme al respecto aquí.

La relación entre la elección de una lengua y la identidad étnica puede ser estrecha, así como es posible que vaya en ambas direcciones: la elección de una lengua dada puede servir como marca de identidad étnica, y una identidad étnica fuerte puede influir en la elección de la lengua que se habla en dicha etnia. En este sentido, los valores del sexto parámetro mencionado anteriormente dicen relación con el hecho de que exista un reconocimiento interno y/o externo de la comunidad lingüística como grupo al interior de la sociedad más amplia que la alberga, así como con la ausencia o presencia de marcas culturales (ya sean éstas objetos o costumbres de significado particular y específico al interior de la etnia): (i) tanto interna como externamente, la comunidad recibe una valoración negativa, y prácticamente no hay marcas culturales, (ii) internamente la valoración positiva es tenue, externamente la valoración es neutral y hay algunas marcas culturales, (iii) internamente la valoración es fuertemente positiva, externamente la valoración es neutral y existen marcas culturales importantes, y (iv) la valoración positiva es fuerte tanto interna como externamente y hay marcas culturales importantes. Esta variable es interesante en el mundo mapuche, por cuanto la valoración, tanto interna como externa, parece haber sufrido cambios positivos importantes en las últimas dos décadas. Está de más decir que la situación varía marcadamente según se trate de hablantes urbanos o rurales, o según se trate de Temuco, Concepción o Santiago, e incluso de qué sectores de esta última ciudad. Los datos de la encuesta son, cuando mucho, indicadores indirectos de las relaciones de valoración actuales.

El prestigio de la lengua es, sin lugar a dudas, un factor que determina de modo importante su viabilidad en el mediano y largo plazo. Los valores del parámetro 7) son: (i) la lengua está desacreditada, (ii) la lengua tiene un estatus neutral, (iii) la lengua es una suerte de lingua franca en la región (o sea, sus propios hablantes nativos y hablantes nativos de otras lenguas la utilizan en contextos religiosos, sociales y/o comerciales), y (iv) la lengua es prestigiosa, reconocida como lingua franca no sólo en la región, sino nacional o internacionalmente. También esta variable ha cambiado favorablemente a lo largo de los últimos veinte años, si bien no puede afirmarse que el mapudungun haya alcanzado los valores (iii) (su valor a la llegada de los europeos, según Luis de Valdivia) o (iv). 
El último parámetro de aquellos discutidos por Landweer, llamado acceso a una base económica estable y aceptable, se refiere al hecho de que una de las causas más comunes de que una comunidad abandone una lengua determinada para adoptar otra es que se cree que dicha adopción será económicamente beneficiosa. Dicho beneficio puede ser percibido o concebido tanto en el corto plazo (necesidades productivas, comerciales o legales inmediatas) como en el largo plazo (lo cual lleva a elegir una lengua prestigiosa para la educación formal, por ejemplo). Los valores de este parámetro son: (i) la comunidad depende por completo de un sistema económico que prescinde de la lengua minoritaria, (ii) existe una economía de subsistencia relativamente marginal que utiliza la lengua minoritaria pero requiere ser complementada por interacción con el sistema económico más amplio, (iii) la comunidad participa de un sistema económico dual en el cual se utilizan la lengua minoritaria y la lengua externa en sus respectivos ámbitos (p. ej., uno agrario y otro industrial), y (iv) la comunidad dispone de un sistema económico estable y aceptable que se sirve de la lengua minoritaria y prescinde de una lengua externa para su funcionamiento. A este respecto, las comunidades de hablantes del mapudungun se encuentran en una posición desfavorable - (i) los urbanos y (ii) al menos algunos de los rurales-, según algunos datos de la encuesta.

\section{Los datos}

No es una exageración decir que las fuentes de información disponibles hasta la fecha acerca de cuántos hablantes tiene el mapudungun no son ni particularmente detalladas ni confiables. Gordon (2005), una de las fuentes comúnmente citadas, da 202.000 hablantes para el mapudungun en Chile en 1982 (incluyendo los dialectos pehuenche y huilliche, este último listado como lengua aparte en el Ethnologue) y 100.000 hablantes en Argentina según estimaciones del año 2000. Un estudio realizado por el CEP en 2002 sugería que el 16\% de quienes se autodefinían como mapuches decía hablar la lengua en algún grado (CEP, 2002: 27). Un estudio del Ministerio de Planificación (Mideplan) arrojaba datos de un orden de magnitud más auspiciosos: un 16,8\% de la población indígena de Chile decía hablar su lengua originaria, mientras que un 18,9\% decía entenderla (Mideplan, 2005: 70). Según veremos a continuación, los resultados de la encuesta del CEP sugieren que el número de hablantes en Chile hoy en día se encuentra entre unas 140.000 y unas 400.000 personas, dependiendo de qué significa ser hablante y de cuántas personas se autoidentifican como mapuches. Antes de discutir estas cifras, sin embargo, veamos datos desagregados respecto de competencia lingüística para poder comprenderlas mejor. 
En el Cuadro № 1-A se aprecia cómo se autoevalúan los encuestados mapuches (M) y no mapuches (NM), tanto aquellos que viven en zonas urbanas (U) como los residentes de zonas rurales (R), en relación con su competencia lingüística. 55 personas de un total de 2.968 declararon hablar el mapudungun mejor que el castellano, de las cuales 53 se identificaron como mapuches, de las cuales a su vez 43 vivían en zonas urbanas. De modo similar, de los 175 encuestados que dijeron hablar ambas lenguas igualmente bien, 162 eran mapuches, la mayoría de ellos (121) rurales. Las cifras comienzan a revertirse en la categoría de quienes hablan la lengua indígena peor que el castellano (168 en total), de los cuales los 139 mapuches se distribuyen casi por partes iguales en términos territoriales: 68 de ellos son urbanos y 71 rurales. 364 personas dijeron entender el mapudungun pero no hablarlo, y en este grupo predominan los mapuches urbanos (204) sobre los rurales (89). Finalmente, aquellas personas que dijeron no entender ni hablar mapudungun ascienden a 2.206, de las cuales la mayor parte son no mapuches (1.370), y en ambos grupos étnicos quienes viven en asentamientos urbanos superan a los rurales (578 vs. 258 y 867 vs. 503, respectivamente). El Cuadro $\mathrm{N}^{\circ} 1$-B expresa estas cifras en términos porcentuales respecto del total de los encuestados.

CUADRO N 1-A: HABLANTES DEL MAPUDUNGUN, SEGÚN COMPETENCIA (ENCUESTADOS)

\begin{tabular}{lrrrrrr}
\hline & MQC & IQC & PQC & ENH & NENH & Total \\
\hline MU & 11 & 41 & 68 & 204 & 578 & 902 \\
MR & 42 & 121 & 71 & 89 & 258 & 581 \\
Total M & 53 & 162 & 139 & 293 & 836 & 1.483 \\
NMU & 0 & 2 & 8 & 26 & 867 & 903 \\
NMR & 2 & 11 & 21 & 45 & 503 & 582 \\
Total NM & 2 & 13 & 29 & 71 & 1.370 & 1.485 \\
Total M+NM & 55 & 175 & 168 & 364 & 2.206 & 2.968 \\
\hline
\end{tabular}

CUADRO N 1-B: HABLANTES DEL MAPUDUNGUN, SEGÚN COMPETENCIA (PORCENTAJES)

\begin{tabular}{lrrrrrr}
\hline & MQC & IQC & PQC & ENH & NENH & Total \\
\hline MU & $0,4 \%$ & $1,4 \%$ & $2,3 \%$ & $6,9 \%$ & $19,5 \%$ & $30,4 \%$ \\
MR & $1,4 \%$ & $4,1 \%$ & $2,4 \%$ & $3,0 \%$ & $8,7 \%$ & $19,6 \%$ \\
Total M & $1,8 \%$ & $5,5 \%$ & $4,7 \%$ & $9,9 \%$ & $28,2 \%$ & $50,0 \%$ \\
NMU & $0,0 \%$ & $0,1 \%$ & $0,3 \%$ & $0,9 \%$ & $29,2 \%$ & $30,4 \%$ \\
NMR & $0,1 \%$ & $0,4 \%$ & $0,7 \%$ & $1,5 \%$ & $16,9 \%$ & $19,6 \%$ \\
Total NM & $0,1 \%$ & $0,4 \%$ & $1,0 \%$ & $2,4 \%$ & $46,2 \%$ & $50,0 \%$ \\
Total & $1,9 \%$ & $5,9 \%$ & $5,7 \%$ & $12,3 \%$ & $74,3 \%$ & $100,0 \%$ \\
\hline
\end{tabular}


A pesar de lo trivial que pudiera parecer, estas cifras no dejan lugar a dudas de que tanto la pertenencia a un grupo étnico como el lugar de asentamiento son factores determinantes en el nivel de competencia lingüística: los mapuches hablan mejor que los no mapuches y las personas de residencia rural hablan mejor que aquellas de residencia urbana. Diferentes tests estadísticos relevantes para evaluaciones de este tipo $\left(\chi^{2}\right.$, análisis de varianza, test exacto de Fisher, métodos basados sobre aleatorización o randomización) arrojan resultados concluyentes en este sentido.

En segundo término, es posible resumir los diferentes valores asignados a la variable "competencia lingüística" de modo de binarizarlos: habla mapudungun vs. no habla mapudungun. Hemos realizado esta simplificación de dos maneras diferentes, a saber: una “dura”, según la cual se considera que sólo los tres primeros valores (habla mejor, habla igual y habla peor que el castellano) corresponden a hablantes, y otra "blanda", según la cual los cuatro primeros valores (o sea, los tres de la interpretación "dura" más aquellos encuestados que dijeron entender el mapudungun pero no hablarlo) lo son. La binarización "dura" pretende dar una medida de la competencia lingüística activa, mientras que la binarización "blanda" es un indicio acerca de la competencia lingüística activa y pasiva. Las cifras agrupadas de esta manera pueden apreciarse en los Cuadros $\mathrm{N}^{\circ} 2$ y $\mathrm{N}^{\circ} 3$, respectivamente.

CUADRO N ${ }^{\circ}$ 2: COMPETENCIA ACTIVA DEL MAPUDUNGUN

\begin{tabular}{lrrrr|r}
\hline & \multicolumn{2}{c}{ CA } & \multicolumn{2}{c}{ SCA } & Total \\
\hline MU & 120 & $13,3 \%$ & 782 & $86,7 \%$ & 902 \\
MR & 234 & $40,3 \%$ & 347 & $59,7 \%$ & 581 \\
Total M & 354 & $23,9 \%$ & 1.129 & $76,1 \%$ & 1.483 \\
NMU & 10 & $1,1 \%$ & 893 & $98,9 \%$ & 903 \\
NMR & 34 & $5,8 \%$ & 548 & $94,2 \%$ & 582 \\
Total NM & 44 & $3,0 \%$ & 1.441 & $97,0 \%$ & 1.485 \\
Total & 398 & $13,4 \%$ & 2.570 & $86,6 \%$ & 2.968 \\
\hline
\end{tabular}

CUADRO N ${ }^{\circ}$ 3: COMPETENCIA ACTIVA Y PASIVA DEL MAPUDUNGUN

\begin{tabular}{lrrrr|r}
\hline & \multicolumn{2}{c}{ CAP } & \multicolumn{2}{c}{ SCAP } & Total \\
\hline MU & 324 & $35,9 \%$ & 578 & $64,1 \%$ & 902 \\
MR & 323 & $55,6 \%$ & 258 & $44,4 \%$ & 581 \\
Total M & 647 & $43,6 \%$ & 836 & $56,4 \%$ & 1.483 \\
NMU & 36 & $4,0 \%$ & 867 & $96,0 \%$ & 903 \\
NMR & 79 & $13,6 \%$ & 503 & $86,4 \%$ & 582 \\
Total NM & 115 & $7,7 \%$ & 1.370 & $92,3 \%$ & 1.485 \\
Total & 762 & $25,7 \%$ & 2.206 & $74,3 \%$ & 2.968 \\
\hline
\end{tabular}


Al igual que con la variable competencia lingüística sin binarizar, tanto en el caso del resumen "duro" como en el del "blando" la pertenencia a grupo étnico y el lugar de residencia son determinantes.

Un resultado poco feliz que se aprecia con claridad en estos cuadros resumidos es el hecho de que sólo una minoría de quienes se autoidentificaron como mapuches dijera ser competente en la lengua: los mapuches que sostienen tener competencia activa son menos de un cuarto (354 de 1.483), e incluso si se considera competencia activa y pasiva son menos de la mitad (647 de 1.483). Si extrapolamos estas cifras a la población total llegamos a una estimación de cuántos hablantes tiene el mapudungun hoy en Chile: según el censo de 2002, 602.677 personas se autoidentificaron como mapuches; por lo tanto, el número de hablantes se localizaría entre 143.862 (competencia activa, 23,9\%) y 262.935 (competencia activa y pasiva, 43,6\%). Si tomamos el dato poblacional de 1992 (muy superior al de 2002, por razones complejas y múltiples: 928.060 mapuches), dichas cifras llegan a 221.533 (23,9\%) y 404.892 (43,6\%) respectivamente.

Las cifras presentadas hasta ahora constituyen elementos que configuran una suerte de fotografía de la situación actual —si bien, naturalmente, deben entenderse sujetas a las limitaciones propias del instrumento, tanto metodológicas como conceptuales. Otros datos recogidos por la encuesta complementan dichas cifras y añaden información que permite especular acerca de la situación futura. Por ejemplo, el Cuadro $\mathrm{N}^{\circ} 4$ resume cifras respecto a con quién aprendieron quienes dicen saber algo de mapudungun (el resto aparece bajo la categoría “residuo”). Obsérvense nuevamente las importantes diferencias entre zonas rurales y aquellas urbanas, así como también entre mapuches y no mapuches. El grueso de estos últimos dice haber aprendido la lengua con conocidos —aun cuando un porcentaje no despreciable, al menos en términos relativos (2,7\%) de no mapuches rurales dice aprenderlo de sus padres-, mientras que la mayoría de los mapuches la ha aprendido con sus padres o abuelos. Nótese que, si se los compara con los abuelos, los padres son mucho más importantes para los mapuches rurales que para los urbanos.

Veamos a continuación dos datos más pertinentes desde la perspectiva de los indicadores de vitalidad etnolingüística esbozados en la sección 2. En primer lugar, se preguntó a los encuestados con qué frecuencia hablaban en lengua mapuche; estos resultados se aprecian en el Cuadro $\mathrm{N}^{\circ}$ 5. Tampoco aquí debería sorprender que los mapuches lo hablen con mayor frecuencia que los no mapuches. Asimismo, en contextos rurales la 
CUADRO N 4: CON QUIÉN SE APRENDE EL MAPUDUNGUN

\begin{tabular}{lrrrrr}
\hline & MU & MR & NMU & NMR & Total \\
& & & & & \\
& & & & & \\
Padres & $10,4 \%$ & $29,7 \%$ & $0,2 \%$ & $2,7 \%$ & $9,6 \%$ \\
Padre/madre & $6,1 \%$ & $8,7 \%$ & $0,1 \%$ & $2,2 \%$ & $4,0 \%$ \\
Abuelos & $9,5 \%$ & $9,3 \%$ & $0,7 \%$ & $1,9 \%$ & $5,3 \%$ \\
Otro pariente & $2,5 \%$ & $2,1 \%$ & $0,3 \%$ & $0,9 \%$ & $1,4 \%$ \\
Conocido & $4,0 \%$ & $2,1 \%$ & $1,7 \%$ & $3,6 \%$ & $2,8 \%$ \\
Profesor & $1,1 \%$ & $0,9 \%$ & $0,4 \%$ & $0,2 \%$ & $0,7 \%$ \\
Otro & $1,6 \%$ & $1,2 \%$ & $0,6 \%$ & $1,7 \%$ & $1,2 \%$ \\
(Residuo & $64,8 \%$ & $46,1 \%$ & $96,0 \%$ & $86,8 \%$ & $74,9 \%)$ \\
Total & $100,0 \%$ & $100,0 \%$ & $100,0 \%$ & $100,0 \%$ & $100,0 \%$ \\
& & & & & \\
\hline
\end{tabular}

lengua indígena se utiliza más frecuentemente que en contextos urbanos. Lo que sí es significativo y preocupante, sin embargo, es el hecho de que quienes dicen usar la lengua a diario sean, aun en el contexto más favorable (mapuches rurales), tan poco numerosos (17,5\% del total; 32,3\% si se desestima el residuo).

El Cuadro $\mathrm{N}^{\circ} 6$ combina los datos de frecuencia con información acerca de los temas que se tratan en lengua mapuche según las declaraciones de los encuestados ( $\mathrm{R}$ representa el residuo). Obsérvese en este contexto lo bajas que son las cifras para temas que deberían mostrar cifras muy superiores si el idioma fuera realmente vital: incluso en el campo, los temas familiares y personales, y aun aquellos relacionados con ceremonias no se

CUADRO N ${ }^{\circ}$ 5: $\quad$ CON QUÉ FRECUENCIA SE HABLA EL MAPUDUNGUN

\begin{tabular}{lrrrrr}
\hline & MU & MR & NMU & NMR & Total \\
\hline A diario & $1,9 \%$ & $17,5 \%$ & $0,1 \%$ & $1,2 \%$ & $4,3 \%$ \\
Varias/semana & $1,7 \%$ & $6,7 \%$ & $0,2 \%$ & $1,4 \%$ & $2,1 \%$ \\
Una/semana & $1,9 \%$ & $3,3 \%$ & $0,2 \%$ & $2,1 \%$ & $1,7 \%$ \\
Varias/mes & $1,7 \%$ & $2,6 \%$ & $0,2 \%$ & $0,2 \%$ & $1,1 \%$ \\
Una/mes & $1,2 \%$ & $2,6 \%$ & $0,1 \%$ & $0,3 \%$ & $1,0 \%$ \\
Varias/año & $4,0 \%$ & $4,1 \%$ & $0,1 \%$ & $0,5 \%$ & $2,1 \%$ \\
Ocasiones esp. & $12,0 \%$ & $9,4 \%$ & $1,4 \%$ & $3,2 \%$ & $6,6 \%$ \\
(Casi) nunca & $11,3 \%$ & $8,7 \%$ & $1,7 \%$ & $4,6 \%$ & $6,6 \%$ \\
(Residuo & $64,5 \%$ & $45,2 \%$ & $95,9 \%$ & $86,5 \%$ & $74,6 \%)$ \\
Total & $100,0 \%$ & $100,0 \%$ & $100,0 \%$ & $100,0 \%$ & $100,0 \%$ \\
& & & & & \\
\hline
\end{tabular}


discuten frecuentemente en mapudungun, sino en castellano. Lo preocupante no es sólo el bajo porcentaje de hablantes en la columna "frecuentemente”, sino especialmente el alto número de hablantes en la columna "nunca”. Incluso si las cifras del Cuadro $\mathrm{N}^{\circ} 6$ reflejan la situación de la comunidad etnolingüística de modo aproximado, el panorama que dibujan sólo puede calificarse de sombrío.

CUADRO N 6: DE QUÉ SE HABLA EN MAPUDUNGUN

\begin{tabular}{|c|c|c|c|c|c|c|c|c|}
\hline & \multicolumn{4}{|c|}{ Mapuches urbanos } & \multicolumn{4}{|c|}{ Mapuches rurales } \\
\hline & Frec. & A veces & Nunca & $\mathrm{R}$ & Frec. & A veces & Nunca & $\mathrm{R}$ \\
\hline Familia & $4,5 \%$ & $13,2 \%$ & $17,4 \%$ & $64,9 \%$ & $16,0 \%$ & $24,4 \%$ & $13,7 \%$ & $46,0 \%$ \\
\hline Pers. & $3,2 \%$ & $10,6 \%$ & $21,3 \%$ & $64,9 \%$ & $16,3 \%$ & $21,2 \%$ & $16,6 \%$ & $45,9 \%$ \\
\hline Salud & $2,4 \%$ & $10,0 \%$ & $22,6 \%$ & $65,0 \%$ & $18,3 \%$ & $16,6 \%$ & $19,2 \%$ & $45,9 \%$ \\
\hline Trabajo & $3,1 \%$ & $7,1 \%$ & $24,6 \%$ & $65,2 \%$ & $18,3 \%$ & $14,4 \%$ & $21,4 \%$ & $45,9 \%$ \\
\hline Deportes & $0,9 \%$ & $6,6 \%$ & $27,5 \%$ & $64,9 \%$ & $9,8 \%$ & $11,0 \%$ & $32,9 \%$ & $46,3 \%$ \\
\hline Política & $0,7 \%$ & $4,3 \%$ & $30,0 \%$ & $65,0 \%$ & $5,7 \%$ & $9,4 \%$ & $38,4 \%$ & $46,5 \%$ \\
\hline Cerem. & $3,8 \%$ & $10,4 \%$ & $20,8 \%$ & $65,0 \%$ & $13,4 \%$ & $20,4 \%$ & $20,0 \%$ & $46,2 \%$ \\
\hline
\end{tabular}

Finalmente, el Cuadro $\mathrm{N}^{\circ} 7$ muestra las cifras más preocupantes de todas: aquellas que dicen relación directa con la transmisión del idioma indígena y, por lo tanto, con su porvenir inmediato. De los mapuches urbanos que dijeron hablar mapudungun, sólo un 2,4\% (0,8\% respecto de 33,2\% $+0,8 \%$ ) dijo hablarlo normalmente con niños pequeños, ya fueran propios o ajenos, en el lugar donde vivía. Incluso los mapuches rurales se comunican predominantemente en castellano con los niños de la zona: sólo un 15,9\% (8,2\% relativo a $43,5 \%+8,2 \%)$ dijo utilizar el mapudungun. En el caso de una lengua vigorosa — piénsese, por ejemplo, en el castellano o el inglés en ciudades como Santiago, Madrid, Londres o Chicago-, estas cifras son iguales a $100 \%$ en comunidades monolingües y ciertamente muy superiores a $70 \%$ en comunidades plurilingües.

CUADRO N 7: $\quad$ EN QUÉ IDIOMA SE HABLA CON NIÑOS PEQUEÑOS

\begin{tabular}{lrr}
\hline & MU & MR \\
\hline Castellano & $33,2 \%$ & $43,5 \%$ \\
Mapudungun & $0,8 \%$ & $8,2 \%$ \\
(Residuo & $66,0 \%$ & $48,3 \%)$ \\
Total & $100,0 \%$ & $100,0 \%$ \\
\hline
\end{tabular}




\section{Conclusiones}

Tanto los datos presentados en la sección precedente como las consideraciones hechas en la sección sobre la vitalidad etnolingüística del mapudungun son alarmantes. Obsérvese que no hay contradicciones: todo apunta en la dirección de una lengua minoritaria que está siendo abandonada por un número sustancial de sus hablantes. La preferencia por la lengua mayoritaria es mayor en ciertos grupos que en otros, así como también en ciertas circunstancias que en otras, lo cual sugiere que, aun cuando las cifras no sean auspiciosas, no nos encontramos aún frente a una lengua claramente moribunda sino a un idioma cuyo estado de salud es crítico — crítico en el sentido original de la palabra griega kritikós, "decisivo”, es decir, cuya sobrevivencia se decidirá en los próximos años, quizás en el transcurso de la próxima generación-. Si no se hace algo que tenga un impacto importante, el pronóstico de Augusta y Coña se hará realidad, y la humanidad habrá perdido una lengua más; Chile continental habrá perdido su último idioma vernáculo en cuanto medio de comunicación vivo y lo verá engrosar las filas de objetos de museo, como los recientemente extinguidos yámana y selknam. Además del castellano (y del inglés), sólo el aimara nortino y el rapa nui insular servirán de recuerdos vivos — ¿ ¿por cuánto tiempo más? - de una época en que la riqueza cultural del territorio chileno, al menos en términos de diversidad biolingüística, era muy superior.

Pero supongamos que suficientes personas no quieren que el mapudungun muera. ¿Qué puede hacerse, y quién debe hacerlo? Un plan de revitalización detallado está más allá de lo que puede cubrir este breve artículo, pero baste aquí mencionar algunas obviedades y acaso algunas cosas menos obvias. El Ministerio de Educación tiene parte de la responsabilidad y de los recursos necesarios para, a través de su Programa de Educación Intercultural Bilingüe, ayudar a revitalizar la lengua mapuche. El próximo perfil sociolingüístico detallado de las regiones VIII, IX y X, así como de la Región Metropolitana, deberá complementar los indicios basados sobre los resultados de la encuesta del Centro de Estudios Públicos que hemos bosquejado aquí, ya sea confirmándolos, matizándolos o corrigiéndolos. Los mapuches urbanos (véase por ejemplo Foerster y Montecino, este volumen), pero especialmente los mapuches rurales, asignan un rol fundamental al mapudungun en el proceso de construcción de la identidad indígena, pero normalmente carecen de la preparación y de los recursos 
necesarios para poder participar en un programa de revitalización, ya sea éste formal o informal, de manera eficaz, sólida, sostenida y competente. El Programa Orígenes y la Corporación Nacional de Desarrollo Indígena deben hacerse cargo de sus respectivas responsabilidades en este sentido. Si bien el Estado ya destina recursos a estas tareas, los serios problemas de legislación, planificación, gestión e implementación que aquejan a la educación pública en Chile son particularmente relevantes para lo que aquí nos ocupa - el aprendizaje formal de la lengua indígena y la formación de profesores de la misma-, por lo que no es de esperar que las dificultades de fondo se resuelvan en el corto plazo sin un esfuerzo considerable por parte de todas las instancias involucradas (véase al respecto el poco alentador panorama descrito en PEIB, 2005). El propósito del presente artículo no es evaluar el desempeño de los programas estatales implementados hasta la fecha; más bien el objetivo es llamar la atención tanto de autoridades como de la opinión pública sobre el hecho de que la vitalidad del mapudungun y, por lo tanto, también su viabilidad, parecen encontrarse severamente amenazadas.

En el contexto de una valoración positiva de la cultura mapuche en Chile por parte de la opinión pública, como se ha venido observando al menos desde comienzos de la década de los noventa, aún queda mucho por hacer, y un reconocimiento amplio del aporte de este pueblo originario podría incluir una mayor presencia escrita de la lengua en las zonas de asentamiento tradicional y la Región Metropolitana (p. ej., más y mejores letreros en mapudungun, tanto de organismos del Estado como de privados, así como señalización de toponimia y caminos). El objetivo de una política tal - como se ha visto en ejemplos análogos de lenguas minoritarias europeas- no sería solamente simbólico sino también práctico: los hablantes aprenderían que la lengua mapuche "sirve para algo”, también en el marco de la sociedad más amplia, y podrían verse motivados a emplearla en ámbitos hasta ahora reservados al castellano. Lo anterior nos lleva a un área aún más delicada, a saber, aquella del aprendizaje informal de la lengua en el ámbito familiar. El gran desafío planteado por la situación actual del mapudungun a los especialistas en educación reside en cómo hacer que los padres decidan hablarlo con sus hijos. Si no se logra este último objetivo, cualquier programa oficial está destinado al fracaso, logrando apenas el mantenimiento del idioma vernáculo como un componente más del folclor o un gabinete de curiosidades. 


\section{BiBLIOGRAFÍA}

Augusta, Fray Félix José de: Gramática Araucana. Valdivia: Imprenta Central J. Lampert, 1903. [Reeditada en 1990 por Ediciones Séneca, Santiago, como Gramática Mapuche Bilingüe].

Brenzinger, Matthias (ed.): Language Diversity Endangered. Berlin: Mouton de Gruyter, 2007.

CEP (Centro de Estudios Públicos): “Estudio Nacional de Opinión Pública N 15, Tercera Serie. Tema Especial: Una Radiografía de los Mapuches”. Documento de Trabajo $\mathrm{N}^{\circ}$ 345. Santiago, 2002.

_ "Estudio de Opinión Pública. Los Mapuche Rurales y Urbanos Hoy, Mayo 2006”. Santiago: CEP, 2006.

Coña, Pascual: Lonco Pascual Coña ñi Tuculpazugun. Testimonio de un Cacique Mapuche. 7. ed. Santiago: Pehuén, 2002.

Crystal, David: Language Death. Cambridge: Cambridge University Press, 2002.

Ferguson, Charles: “Diglossia”. Word 15, 1959.

Fishman, Joshua (ed.): Can Threatened Languages Be Saved? Clevedon: Multilingual Matters, 2001.

Gordon, Raymond (ed.): Ethnologue: Languages of the World. Fifteenth edition. Dallas: SIL International, 2005. [En Internet: http://www.ethnologue.com/]

Grenoble, Lenore y Lindsay Whaley (eds.): Endangered Languages: Language Loss and Community Response. Cambridge: Cambridge University Press, 1998.

Grenoble, Lenore y Lindsay Whaley: Saving Languages: An Introduction to Language Revitalization. Cambridge: Cambridge University Press, 2005.

Hinton, Leanne y Kenneth Hale (eds.): The Green Book of Language Revitalization in Practice. New York: Academic Press, 2001.

Kindell, Gloria y Paul Lewis (eds.): Assessing Ethnolinguistic Vitality: Theory and Practice. Selected Papers from the Third International Language Assessment Conference. Dallas: SIL, 2000.

Landweer, Lynn: "Schlie-Landweer Priority Allocation Assessment Device: Rationale Paper”. En Gloria Kindell (ed.): Proceedings of the Summer Institute of Linguistics International Assessment Conference, Horseleys Green, 23-31 May 1989, pp. 49-67. Dallas: SIL, 1991.

- "Endangered Languages. Indicators of Ethnolinguistic Vitality". Notes on Sociolinguistics 5.1, 2000.

Mideplan (Ministerio de Planificación): Encuesta de Caracterización Socioeconómica Nacional y Población Indígena CASEN 2003. Santiago, 2005.

Moseley, Christopher: Encyclopedia of the World's Endangered Languages. Oxford: Curzon Press, 2007.

Nettle, Daniel \& Suzanne Romaine: Vanishing Voices. The Extinction of the World's Languages. Oxford: Oxford University Press, 2002.

PEIB (Programa de Educación Intercultural Bilingüe): Descripción del Contexto Sociolingüístico en Comunidades Indígenas de Chile. Santiago de Chile: Ministerio de Educación, 2005.

Tsunoda, Tasaku: Language Endangerment and Language Revitalization. Berlin: Mouton de Gruyter, 2005.

Valdivia, Luis de: Arte y Gramatica General de la Lengva que Corre en Todo el Reyno de Chile, con vn Vocabulario, y Confessionario. Lima: Francisco del Canto, 1606. 